\title{
Hereditary episodic ataxia
}

INSERM

\section{Source}

INSERM. (1999). Orphanet: an online rare disease and orphan drug data base. Hereditary episodic ataxia. ORPHA:211062

Hereditary episodic ataxia (EA) represents a group of neurological disorders characterized by recurrent episodes of ataxia and vertigo which may be progressive. Weakness, dystonia and ataxia are sometimes present in the interictal period. Seven types of EA have been described to date (EA type 1 to EA type 7, see these terms), but most of the reported cases belong to EA1 and EA2. 